\title{
A COMPARISON OF THE COMPOSITION OF NEW AND EVOLVED COMETS
}

\author{
B. DONN
}

The intensity ratio of the continuum to the molecular emissions was estimated in the spectra of eight-five comets. These consisted of 34 new, 1/a (orig) $<100 \times 10^{-6} \mathrm{AU}^{-1}, 31$ more evolved with $10^{2}<1 / \mathrm{a}<10^{4}$, and 20 periodic comets, $P<10^{3}$ years. In each age group the comets were divided into two distance intervals, the first observed at less than 1 AU from the sun and the second category observed at more than $I \mathrm{AU}$. No significant difference among the patterns of relative intensity distribution among four age groups was found. No general evidence for a difference between pre- and post-perihelion observations was found although in a few cases a pronounced effect occurs. Four conclusions are drawn. (1) There is no readily apparent difference in continuum to emission intensity ratio between new and more evolved comets. (2) An intrinsic distribution of this characteristic does occur. (3) Periodic comets with weak continua derived from new comets with the same property. (4) No weakening of the continuum in general occurs following perihelion passage. The infrared evidence for Comet Encke suggests that the faintness of its continuum may be caused by a size distribution containing only particles larger than about $10 \mathrm{\mu m}$.

In this paper I examine some evidence on the original composition of comets and implications of the study.

It was pointed out about twenty years ago and now generally, although not completely accepted, that comet nuclei are relatively unchanged samples of the primordial material from which they formed. It is also commonly believed that their origin was part of the origin of the solar system. Recently, there have been several proposals (Cameron 1973; Donn 1976) that comets formation, although associated with the origin of the solar system, occurred in interstellar clouds at distances corresponding to that of the Oort cloud.

A determination of the original composition of comets before modification by near solar approaches would reveal a fundamental characteristic of comets. Such data would be a most significant clue to the composition of the cloud from which comets formed and may be a reliable indicator of the place of formation, whether primordial solar nebula or interstellar cloud. In this paper l examine the continuum to molecular emission intensity ratio in cometary spectra as an indicator of initial composition. This ratio presumably measures the dust to gas ratio in comets or more precisely, the component of gas yielding visible emissions.

The condition of looking at comets that have made no or very few previous solar approaches means we are concerned with what Oort (1950) called new comets. 
These are objects which according to the comet origin hypotheses adopted here have been in the Oort cloud for about $5 \times 10^{9}$ years. During this interval they have been exposed to the galactic radiation environment (Shul'man 1972; Donn 1976; Whipple 1976).

The composition of these objects was first investigated by Oort and schmidt in 1950 and has not been examined since. Orbital analyses have greatly improved in the last quarter century and many additional comets with nearly parabolic. orbits have been observed in that period. Consequently, the data on spectra of new comets is both more extensive and more reliable. It is therefore desirable to reexamine relationships between spectra and composition of 'new' and more evolved comets. This is particularly desirable as some questions can be raised about Oort's interpretation of some of the associate phenomena.

The criterion adopted for new comets is a $>10^{4}$ A.U. or $1 / a<100 \times 10^{-6}$ A.U. -1 where a is the semimajor axis. For 32 comets with $q>2.2$ for which nongravitational effects seem to be negligible Marsden and Sekanina (1973) obtained a mean error in $1 / a$ of $44 \times 10^{-6}$. Marsden (1976) takes $1 / \mathrm{a}<200 \times 10^{-6} \mathrm{~A}$.U. 1 as an indication of a new comet or one with only two or three returns to the sun. For all long period comets in Marsden's (1975) catalogue for which he gave osculating 1/a values, the planetary perturbations of Everhart and Raghavan (1970) were applied to obtain original $1 / a$ values $i . e .$. for the orbit at large heliocentric distances where planetary perturbations had not yet had any affect. In a few cases Marsden (1976) made available more recent calculations for new comets. In this way a catalogue of new comets from 1853 through 1975 was obtained.

The literature was searched for spectra of these comets. The most extensive list of references is Bobrovnikov's (1942) bibliography of spectra. A few extensive descriptions of cometary spectra exist (Baldet 1972; Bobrovnikov 1927; Swings and Haser 1957). When this task was nearly complete, it was realized that a comparison with the characteristics of spectra of evolved comets would be necessary. Older comet spectra were added in a somewhat selective, more or less random manner, to yield a sufficiently extensive set for a meaningful comparison among new and several classes of older comets. I plan to continue this study of cometary spectra to make it as complete as possible. Publication of an annotated bibliography is also planned.

This work was motivated by an impression that cometary spectra fell into three broad general categories: (1) continuum dominated, neutral molecular and ion emission relatively weak; (2) molecular emissions dominate, continuum relatively weak or very narrow; (3) ionic emission, notably $\mathrm{CO}^{+}$, dominates, other two components much weaker. These three types seemed to apply to both new and older comets in contradiction to Oort's conclusion.

The spectra or their descriptions were examined only from this point of view. All spectra were classified according to the relative intensity ratio of the continuum to molecular emissions. Considerable precaution is needed in attempting this procedure. Oort (1951) discussed one of the major problems, the strong tendency of the relative intensity of the continuum to increase with heliocentric distance. This is demonstrated in Figure 1, taken from Swings and Haser (1957). The numbers at the right are the heliocentric distance. It will be shown here, however, that he did not properly allow for this effect. Care is also necessary for comparing the strength of the continuum in spectra taken with different instruments. Low dispersion slit spectra and objective prism spectra make the continuum appear stronger. This characteristic is also illustrated by Swings and Haser (1957). Because of these difficulties and the qualitative data of nearly all spectra, only three categories of continuum to emission intensity ratio were used; high, medium and low. High means continuum dominates, medium that continuum and emission are each prominent and low means the continuum is weak or absent.

There is believed to be little uncertainty in the high and low categories. 


\section{NEW AND EVOLVED COMETS}

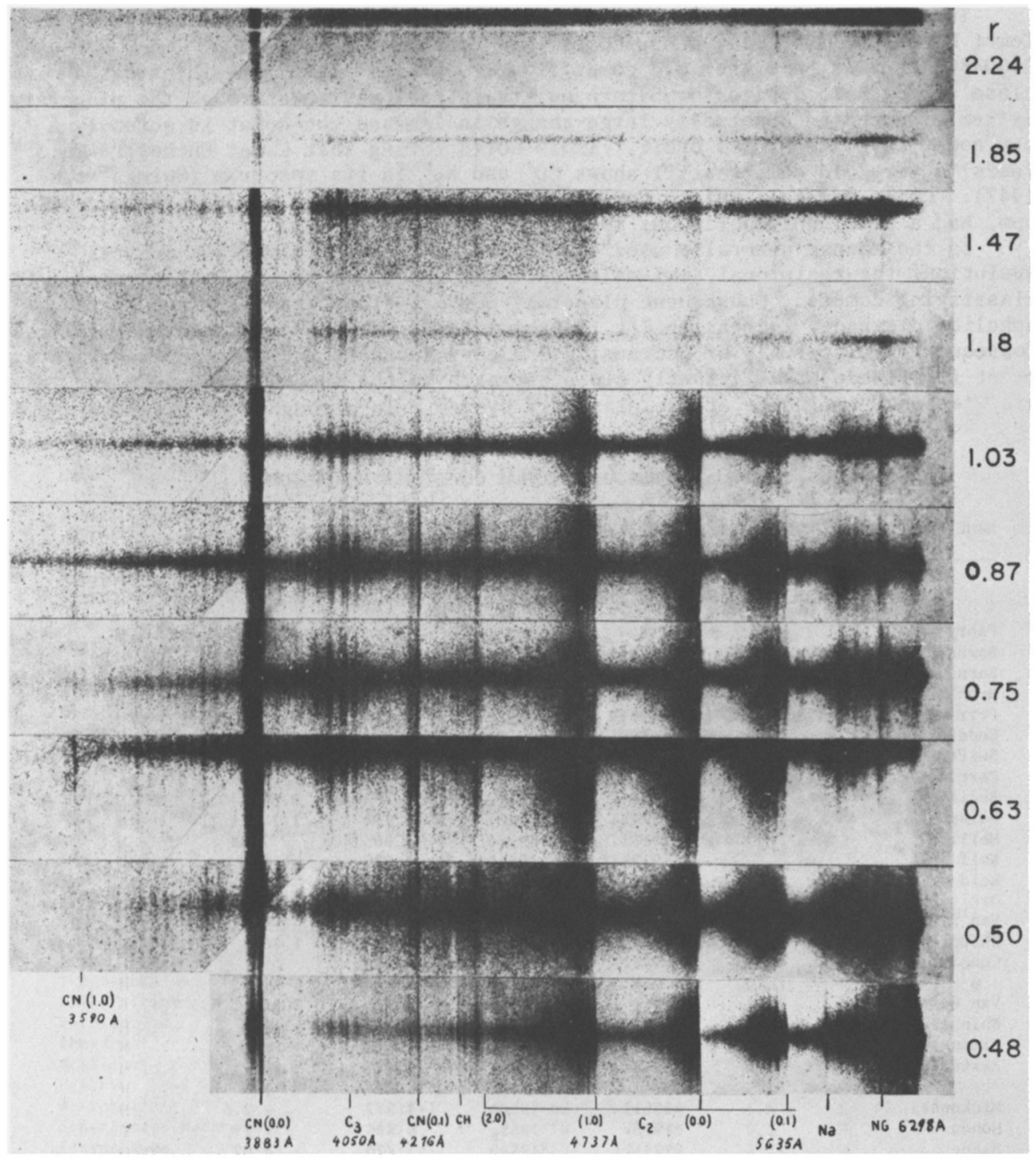

rigure 1. pre-perihetion spectra of Comet Cunningham $1941 \mathrm{I}$. The numbers are the heliocentric distances at time of observations.

For the medium group the classification is more uncertain and arbitrary. It includes those spectra for which an unambiguous assignment was difficult. This occurred because only ambiguous verbal descriptions were available or different spectra indicated possible different classifications. In many cases the spectra clearly seemed to fall into the medium intensity ratio category. In Figure 1 , spectra for $r$ greater than 1.03 AU represent high continuum to emission ratios, $\mathbf{r}=0.87-0.63$ are examples of the medium group and the last two fall in the low continuum categdry. 
There are only two comets in which the $\mathrm{CO}^{+}$spectra is dominant. These are Comet Morehouse 1908 III, a new comet, and Comet Humason, 1962 VIII, $1 / a=$ 0.00489 , that is, a rather old comet from its orbit. It is possible that a close approach to Jupiter or Saturn on its initial passage through the planetary system produced an abnormally large change in 1/a and the comet is actually a new comet in spite of its orbit. It is worth noting that Comet Encke, $P=3.3$ years, a very old comet, still shows $\mathrm{Co}^{+}$and $\mathrm{N}_{2}^{+}$in its spectrum (Swings et al. 1947). Comet Humason, unlike Comet Morehouse which showed no detectable continuum, had a prominent continuous spectrum (Greenstein 1962).

In the manner generally used in studies of the Oort cloud and orbital evolution, the reciprocal semi-major axis was chosen as the orbital parameter for classifying comets. Subsequent planetary perturbations gradually reduce the aphelion distance, increasing 1/a (Everhart 1976; Marsden and Sekanina 1973). Consequently, intervals of increasing $1 / a$ correspond to increasing time since a comet first made a sufficiently close approach to the sun to come under the gravitational influence of the planetary system. In a rough statistical sense,

TABLE I

ORBITAL CLASSES AND SPECTRAL CLASSIFICATION OF COMETS

\begin{tabular}{|c|c|c|c|c|c|}
\hline \multirow[t]{2}{*}{ NAME } & \multicolumn{2}{|c|}{ DESIGNATION } & \multirow[t]{2}{*}{$q(A . U)}$. & \multirow[t]{2}{*}{$\mathbf{r}(A . U)}$. & $\begin{array}{l}\text { SPECTRAL } \\
\text { CLASS } \\
\end{array}$ \\
\hline & $1 / a<100$ & $\mathrm{O}^{-6} \mathrm{Au}^{-1}$ & & & \\
\hline Fabry & $1886 \mathrm{I}$ & $1885 \mathrm{~d}$ & 0.642 & $0.65-1$ & $\mathrm{M}$ \\
\hline Barnard-Hartwig & $1886 I X$ & $1886 \mathrm{f}$ & 0.663 & $\sim 1$ & $M$ \\
\hline Barnard & $1889 \mathrm{I}$ & $1888 \mathrm{e}$ & 1.85 & -2 & $\mathrm{H}$ \\
\hline Brooks & 189011 & $1890 \mathrm{a}$ & 1.908 & 1.9 & $\mathrm{H}$ \\
\hline Perrine & $1895 \mathrm{IV}$ & $1895 \mathrm{c}$ & 0.192 & $0.35-0.95$ & $\mathrm{H}$ \\
\hline Coddington & I 898V I I & $1898 \mathrm{c}$ & 1.702 & 2.2 & $\mathrm{H}$ \\
\hline Swift & $1899 \mathrm{I}$ & 1899a & 0.327 & $0.7-1.3$ & L \\
\hline Perrine & 1902 III & $1902 \mathrm{~b}$ & 0.401 & 0.85 & $\mathrm{~L}$ \\
\hline Beliavsky & $1911 \mathrm{IV}$ & $191 \mathrm{Ig}$ & 0.303 & $0.31-0.37$ & L \\
\hline Delavan & $1914 \mathrm{~V}$ & $3913 \mathrm{f}$ & 1.104 & $1.1-1.2$ & M \\
\hline Mellish & $1915 \mathrm{II}$ & $1915 a$ & 1.005 & 2.24 & M \\
\hline Wolf $f$ & 1917 III & $1916 \mathrm{~b}$ & 1.686 & 1.9 & $\mathrm{H}$ \\
\hline Reid & $1921 \mathrm{II}$ & $1921 a$ & 1.008 & -1 & L \\
\hline Orkisz & $1925 \mathrm{I}$ & $1925 \mathrm{c}$ & 1.109 & 1.2 & L \\
\hline Van Biesbroeck & $1925 V I I$ & $1925 \mathrm{i}$ & 1.506 & $>1.5$ & L \\
\hline Whipple & 1937 IV & $1937 \mathrm{~b}$ & 1.734 & $1.76-1.86$ & $\mathrm{H}$ \\
\hline Cumningham & 19411 & $1940 \mathrm{c}$ & 0.368 & $\begin{array}{l}5 \\
1.5\end{array}$ & $\begin{array}{l}\mathrm{L} \\
\mathrm{H}\end{array}$ \\
\hline Van Gent & 1941VIII & $1941 \mathrm{~d}$ & 0.875 & $1.3-1.6$ & L \\
\hline Whipple-Bernasconi-Kul in & $19421 \mathrm{~V}$ & $1942 \mathrm{a}$ & 1.445 & 1.7 & $\mathrm{H}$ \\
\hline Timmers & $1946 \mathrm{I}$ & $1946 a$ & 3.725 & $>1.7$ & M \\
\hline \multirow[t]{2}{*}{ Bester } & $1948 \mathrm{I}$ & $1947 \mathrm{k}$ & 0.748 & 0.8 & M \\
\hline & & & & 1.3 & $\mathrm{H}$ \\
\hline Minkowski & $1951 I$ & $1950 \mathrm{~b}$ & 2.572 & $>2.6$ & $\mathrm{H}$ \\
\hline Honda & $1955 \mathrm{~V}$ & $1955 \mathrm{~g}$ & 0.885 & $<1$ & $\mathrm{~L}$ \\
\hline Baade & $1955 \mathrm{VI}$ & $1954 \mathrm{~h}$ & 3.870 & 4.02 & CONT. $-\mathrm{H}$ \\
\hline Arend-Rol and & 1957 I I I & $1956 \mathrm{~h}$ & 0.316 & 0.55 & $\mathrm{H}$ \\
\hline Burnham & $19601 \mathrm{I}$ & $1959 \mathrm{k}$ & 0.504 & $0.77-1.09$ & $\ddot{L}$ \\
\hline Seki-Lines & $19621 \mathrm{II}$ & $1962 c$ & 0.031 & 0.55 & H \\
\hline Kilston & $1966 \mathrm{~V}$ & $1966 \mathrm{~b}$ & 2.385 & 2.4 & $\mathrm{~L}$ \\
\hline Rudnicki & 1967 I I & $1966 \mathrm{e}$ & 0.429 & 1.04 & $\mathrm{~L}$ \\
\hline Honda & $1968 \mathrm{VI}$ & $1968 \mathrm{c}$ & 1.160 & 1.2 & M \\
\hline Heck-Sause & 1972 VII I & $1973 a$ & 2.511 & 2.73 & $\mathrm{H}$ \\
\hline Kohoutek & $1973 \times 11$ & $1973 \mathrm{f}$ & 0.142 & $<1$ & $M$ \\
\hline \multirow[t]{2}{*}{ Bradfield } & & $1975 d$ & 1.2 & $>1.2$ & M \\
\hline & $100<1 / a$ & & & & \\
\hline Wells & $1882 \mathrm{I}$ & $1882 a$ & 0.061 & 0.4 & L \\
\hline Morehouse & 1908 I I I & $1908 c$ & 0.945 & 0.95 & $\mathrm{CO}+-\mathrm{L}$ \\
\hline Great & $1910 I$ & $1910 a$ & 0.129 & 0.3 & $\mathrm{H}$ \\
\hline Finsler & $1937 \mathrm{~V}$ & $1937 f$ & 0.863 & 0.9 & L \\
\hline
\end{tabular}


$200<1 / \mathrm{a}<1000$

Barnard
Brooks
Stearns
Honda-Bernasconi
Barbon
Ikeya-Seki
Tago-Sato-Kosaka
Bradfield
Kobayashi-Berger-Milon

\section{Coggia}

Tebbutt (Great)

Daniel

Kriess

Brooks

Quenisset

Gale

Peltier

Kosik-Peltier

Jurlof-Achmarof-Hasse 1

deKock-Paraskevopoulos

Whipple-Fedtke

Great Southern

Eclipse

Mrkos

Alcock

Bennet t

West

$\begin{array}{ll}1886 \text { I I } & 1885 \mathrm{e} \\ 1904 \mathrm{I} & 1904 \mathrm{a} \\ 1927 \mathrm{IV} & 1927 \mathrm{~d} \\ 1948 \mathrm{IV} & 1948 \mathrm{~g} \\ 1966 \mathrm{I} & 1966 \mathrm{c} \\ 1968 \mathrm{I} & 1968 \mathrm{n} \\ 1969 \mathrm{IX} & 1969 \mathrm{~g} \\ 1974 \mathrm{III} & 1974 \mathrm{~b} \\ & 1975 \mathrm{~h}\end{array}$

$1000<1 / \mathrm{a}<10,000$

$\begin{array}{ll}1874 \text { I I I } & 1874 \mathrm{c} \\ 1881 \text { III } & 1881 \mathrm{~b} \\ 1907 \text { IV } & 1907 \mathrm{~d} \\ 1911 \text { II } & 1911 \mathrm{~b} \\ 1911 \mathrm{~V} & 1911 \mathrm{c} \\ 1911 \mathrm{VI} & 1911 \mathrm{f} \\ & \\ 1912 \mathrm{II} & 1912 \mathrm{a} \\ 1936 \text { II } & 1936 \mathrm{a} \\ 1939 \mathrm{I} & 1939 \mathrm{a} \\ 1939 \mathrm{III} & 1939 \mathrm{~d} \\ 1941 \mathrm{IV} & 1941 \mathrm{c} \\ 1943 \mathrm{I} & 1942 \mathrm{~g} \\ 1947 X I \mathrm{I} & 1947 \mathrm{n} \\ & \\ 1948 X \mathrm{I} & 19481 \\ & \\ 1957 \mathrm{~V} & 1957 \mathrm{~d} \\ 1963 \mathrm{III} & 1963 \mathrm{~b} \\ 1970 \text { II } & 1969 \mathrm{i} \\ & 1975 \mathrm{n}\end{array}$
0. 479
2.7018
3.684
0.208
2.018
1. 697
0.473
0.503
0.426

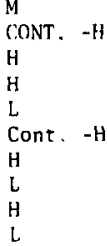

0.676

0.735

ก. 512

0.684

0.489

0.788

0.716

1.100

0.717

0.528

0. 790

1.353

0.110

0.135

0.355

1.537

0.538

0.197

0.9
2.4
2.74
3.7
$67-0.86$
2.4
1.73
$0.49-0.83$
0.6
$<1.0$

PERIODIC COMETS

Name

Seki

Ikey a-Seki

Wilk-Peltier

Perrine

Ikeya

Mellish

Halley

Westphal

Schwassmann-Wachmann $I^{2}$

Kearns-Kwee

Schaumasse

Faye

Giacobini-Zinner

deVico-Swift

Kopff

Pons-Winnecke

d'Arrest

Tutt le-Giacobini-Kresak

Temple II

Encke

Designation ${ }^{1}$

q(A.U.) r(A.U.) Spectra1 Class

\begin{tabular}{|c|c|c|c|c|}
\hline I96IVIII & 942 & 0.681 & $<1$ & Pure Emission - L \\
\hline $1965 \mathrm{VIII}$ & 850 & 0.008 & 0.01 & Sun Grazer - M \\
\hline 1937 I I & 589 & 0.620 & 0.65 & $L$ \\
\hline 18981 & 417 & 1.095 & $>1.1$ & $M$ \\
\hline 1964 V I I I & 363 & 0.822 & 0.85 & $\mathbf{M}$ \\
\hline $1917 \mathrm{I}$ & 145 & 0.190 & $<1$ & $\mathrm{~L}$ \\
\hline $1910 I I$ & 76 & 0.587 & $<1$ & $M$ \\
\hline $1913 \mathrm{VI}$ & 62 & 1.254 & 1.5 & $\mathbf{L}$ \\
\hline 1974 & 15 & 5.448 & 5.5 & $\mathrm{H}$ \\
\hline $1972 \times I$ & 8.9 & 2.229 & 2.25 & $\mathrm{H}$ \\
\hline 1960 I I I & 8.2 & 1.196 & 1.25 & M \\
\hline $1969 \mathrm{VI}$ & 7.4 & 1.616 & 1.93 & $H$ \\
\hline $1972 \mathrm{VI}$ & 6.4 & 0.994 & 1 & $\mathrm{H}$ \\
\hline 1965 VII & 6.3 & 1.625 & 1.65 & $\mathrm{H}$ \\
\hline $1970 \times I$ & 6.3 & 1.567 & 1.66 & $L$ \\
\hline 1970 VI I I & 6.3 & 1.247 & $>1.25$ & $H$ \\
\hline $1976 \mathrm{e}$ & 6.2 & 1.167 & -1.2 & $M$ \\
\hline $1973 V I$ & 5.5 & 1.152 & $>1.2$ & $M$ \\
\hline $1972 X$ & 5.3 & 1.364 & $>1.4$ & $\mathrm{H}$ \\
\hline $1974 \mathrm{~V}$ & 3.3 & 0.338 & $<1$ & Pure Emission - L \\
\hline
\end{tabular}

1 last recorded apparition

2 observed at outburst 
the age of a comet varies inversely as $1 / a$. The first group $1 / a<100 \times 10^{-6}$ are the new comets. A few probably new comets with $1 / a<200 \times 10^{-6}$, are included in the second group. Comets in each of the four intervals have been separated according to the distance at which the spectra were obtained, the dividing line being $1 \mathrm{AU}$. In a few cases for the earliest spectra, the perihelion distance was used.

The results are tabulated in Table $I$ and shown in Figure 2 in the form of histograms for each orbital or age group. In each of the four groups the first three columns, shaded gray, are the distribution of intensity ratios obtained within $1 \mathrm{AU}$. The second set of three columns, filled with dashes, show the distribution for comets observed beyond $1 \mathrm{AU}$. It is seen that each orbital or age group has a similar general pattern of continuum to emission intensity ratios. It is particularly noteworthy that the patterns for new and for periodic comets are very similar in spite of the considerable age, short periods and consequent highly evolved character of the periodic comets. Unless the sample plotted here is very biased, it seems well established that there is little change in the spectrum of comets with evolution. A preponderance of strong or weak continua spectra is a function of heliocentric distance at time of observation. In every one of the four intervals strong molecular emission dominates within 1 $\mathrm{AU}$ and strong continua dominates beyond $1 \mathrm{AU}$.

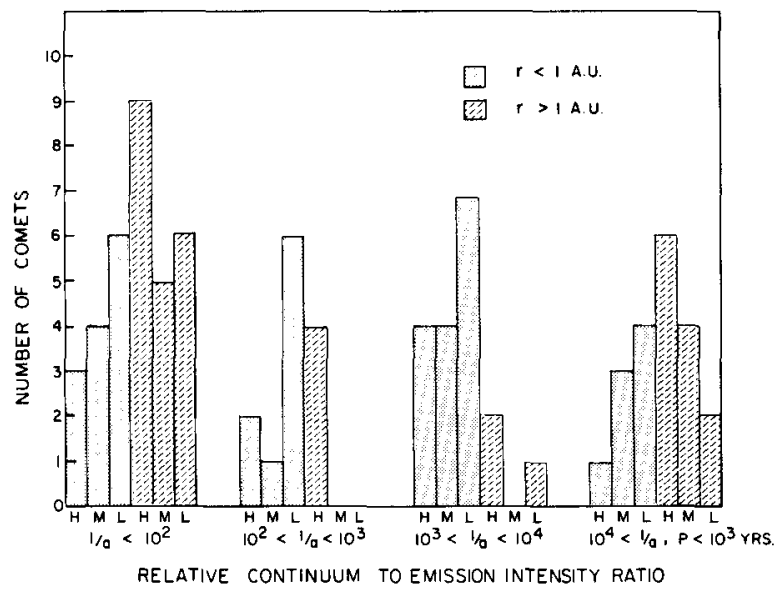

Figure 2. Histogram of continuum to emission intensity ratios for four age groups. The three dark gray blocks on the left of each group are for comets observed at less than I AU from the sun, the three light gray blocks on the right are for comets observed beyond 1 AU. $H=$ high, $M=$ medium and $L=$ low values of the ratio.

Oort and Schmidt (1951) concluded from their analysis of photometric data that there is a considerable difference between new and older comets in the dependence of luminosity upon heliocentric distance. If we repeat their analysis and again separate the data into two groups, perihelion distance $q<1.5 \mathrm{AU}$ and $\mathrm{q}$ greater than $1.5 \mathrm{AU}$, the difference disappears. A separation at $1.5 \mathrm{AU}$ was adopted because Figure 2 of their paper has a gap at that distance. Their expression for luminosity is

$$
\mathrm{m}^{\prime}=\mathrm{A}+\mathrm{Br}^{1 / 2}
$$

When $\mathrm{m}^{\prime}$ is the luminosity reduced to a geocentric distance of $1 \mathrm{AU}$ and $\mathrm{r}$ is the 
heliocentric distance. The parameter B measures the rate of change of luminosity with heliocentric distance. For the comets studied by Schmidt, the B values for the two groupings of Oort and Schmidt, new plus almost new comets and old plus periodic comets, are given in Table II.

TABLE II

COMPARISON OF B VALUES

$$
m^{\prime}=A+B r^{1 / 2}
$$

\begin{tabular}{|c|c|c|c|c|c|c|}
\hline \multirow[b]{2}{*}{ Category } & \multicolumn{3}{|c|}{$q<1.5$} & \multicolumn{3}{|c|}{$q>1.5$} \\
\hline & no. & $\overline{\mathbf{B}}$ & m.e. & no. & $\overline{\mathrm{B}}$ & m.e. \\
\hline new, almost new & 19 & 7.4 & 0.7 & 15 & 2.4 & 0.3 \\
\hline old, periodic & 24 & 8.5 & 0.7 & 2 & 4.6 & 1.4 \\
\hline
\end{tabular}

Under each distance interval the successive colunns give the number of comets, the mean value of $B$ and its mean error. Again we see that within each distance interval the average $B$ for each orbital group shows no significant difference whereas within an orbital group the average $B$ has a large difference in going from the nearer to the more distant comets. This result is consistent. with the well established small heliocentric luminosity index for the continuum compared to a much larger index for the molecular emissions.

The present study leads to several significant conclusions. (1) There is no evidence for a difference in the continuum to emission ratio between new and more evolved comets. (2) Among all age groups an intrinsic spread of that ratio is found. (3) The similarity of the continuum to emission ratio among all orbital or age groups suggests that no general systematic change occurs in this characteristic as comets evolve from new to periodic.

Some further comments on the present analysis and conclusions will now be made. There are a few well observed instances of the continuum of a comet fading appreciably after perihelion. Comet Kohoutek is a notorious recent example (A'Hearn and Cowan 1975). Another example is the new sun grazer, Comet Seki-Lines 19621II, $q=0.03$ AU (Greenstein 1962, warner 1962). On the other hand, Comet Ikeya-Seki 1965 VIII, $q=0.008 \mathrm{AU}$, had a very spectacular dust tail after perihelion and the same was true of Comet Bennett. An examination of new comets in Table I observed within I AU shows all three ratio classes scattered rather evenly between pre- and post-perihelion observations. In several cases other than those cited, spectra were observed at both intervals with no difference. The similarity of the patterns among all age classes in Figure 1 strongly supports the conclusion that perihelion passage does not produce a general weakening of the continuum. A fourth conclusion thus may be added. (4) There is no general decrease of the continuum intensity after perihelion al though occasionally that does occur.

Comet Encke is a particularly interesting object which has been studied as an example of a highly evolved comet. Delsemme and Rud (1973) suggest that its nucleus is only partially covered with ices. Sekanina (1972) proposed that Comet Encke now consists of a porous core from which volatiles diffuse out to produce a coma as perihelion is approached. Comet Encke also has an extremely weak, photographically undetectable continuum (Swings 1947; Liller 1965). As a result of this analysis I propose that periodic Comet Encke is derived from the class of new comets that have weak continua. It seems likely that the result of many close solar passages during its orbital evolution further reduced the intensity of the continuum. However, there are the other periodic comets listed 
in Table I which still possess a very prominent continuum, including several observed within or near 1 AU. Swings (1947) also called attention to the close similarity between the emission spectra of Comet Encke and of comets of long period. There is no obvious difference in the molecular components except for the weakness but not complete absence of $\mathrm{CO}^{+}$and $\mathrm{N}_{2}^{+}$in the spectrum of Encke.

A more detailed investigation of the molecular emission features in new and in evolved would appear to be very informative. Such a study will be carried out as part of this program.

An additional significant characteristic of Comet Encke is the presence of a prominent infrared continuum, such that the ratio of infrared luminosities of Comets Encke and Bennett (Ney 1976) is roughly proportional to their Lyman- $\alpha$ luminosities (Bertaux et a1. 1973; Keller 1976). Consequently, Encke does show evidence of a significant dust coma. The absence of visible scattering can be explained by the occurrence of particles larger than $10 \mu \mathrm{m}$. For particles greater than that size the scattering efficiency is less than one percent of that at $1 \mu \mathrm{m}$ (Greenstein 1951). Such grains would not show that $10 \mu \mathrm{m}$ emission feature because they would be optically thick (Ney 1976). Unfortunately, because of the low $10 \mu \mathrm{m}$ luminosity of Comet Encke, Ney was unable to obtain any measurements there or at longer wavelengths. The suggestion from such data is that the continuum to emission ratio may measure particle size distribution rather than the relative abundance of solid grains. Until infrared measurements on many more comets become available the validity of the suggestion just made will be uncertain.

\section{ACKNOWLEDGEMENTS}

Much of the time consuming work of obtaining the references to spectroscopic observations was done by Ronald Ticker. He also assembled Table I and assisted with Figure 2. Dr. Regina Cody also assisted in the collection and classification of the spectra.

\section{REFERENCES}

A'Hearn, M., and Cowan, J. J. 1975, Astron. J., 80, 852.

Baldet, F. 1927, Ann. Obs. Paris, 7, 1.

Bertaux, J. L., Blamont, J. E., and Festou, M. 1973, Astron. Astrophys., 25, 415.

Bobrovnikov, N. T. 1927, Astroph. J., 66, 439.

Cameron, A.G.W. 1973, ICarus, $18,407$.

Delsemme, A. H., and Rud, D. A. 1973, Astron. Astrophys. , 28, 1.

Donn, B. 1976, in B. Donn, M. Mumma, W. Jackson, M. A'Hearn and R. Harrington (eds.), The study of Comets, NASA SP-393, NASA Washington, D.C., p. 663.

Donn, B. 1976, in B. Donn, M. Mumma, W. Jackson, M. A'Hearn and R. Harrington (eds.), The Study of Comets, NASA SP-393, NASA Washington, D.C., p. 611.

Everhart, E. 1976, in B. Donn, M. Mumma, W. Jackson, M. A'Hearn and R. Harrington (eds.), The Study of Comets, NASA SP-393, NASA Washington, D.C., p. 445.

Everhart, E., and Raghavan, N. 1970, Astron. J., 75, 258.

Greenstein, J. L. 1951, in J. A. Hynek (ed.), Astrophysics, McGraw Hill Co., N.Y., Ch. 13, p. 526.

Greenstein, J. L. 1962, Astrophys, J., 136, 589.

Keller, H. W. 1976, in B. Donn, M. Mumma, W. Jackson, M. A'Hearn, and R. Harrington, (eds.), The Study of Comets, NASA SP-393, NASA Washington, D.C., p. 287.

Liller, W. 1965, Astron. J., 66, 372.

Marsden, B. G. 1975, Catalogue of Cometary Orbits, 2nd ed., Central Bureau for Astron. Telegrams, Smithsonian Astrophysical Obs., Cambridge, Mass. 
Marsden, B. 1976, in preparation.

Marsden, B. G., and Sekanina, Z. 1973, Astron. J., 78, 1118.

Ney, E. 1976, in B. Donn, M. Mumma, W. Jackson, M. A'Hearn, and R. Harrington (eds.), The study of Comets, NASA SP-393, NASA Washington, D.C., p. 334.

Oort, J. H. 1950, Bull. Ast. Inst. Neth., 11, 91 .

Oort, J. H., and Schmidt, M. 1951, Bul1. Ast. Inst. Neth. , 11, 259.

Sekanina, Z. 1972, in G. A. Chebatarev, E. I. Kazimirchak-Polonskaya and B. G. Marsden, (eds.), The Motion, Evolution of orbits and Origin of Comets, D. Reidel Pub. Co., Dordrecht, Holland, p. 301.

Shul'man, L. M. 1972, in G. A. Chebatarev, E. I. Kazimirchak-Polonskaya and

B. G. Marsden, (eds.), The Motion, Evolution of Orbits and Origin of comets, D. Reidel Pub. Co., Dordrecht, Holland, p. 265.

Swings, P. 1947, Ann. Astrophys., 11, 124.

Swings, P., and Haser, L. 1957, Atlas of Cometary spectra, Liege, 1957.

Whipple, F. L. 1977, this volume. 